\title{
Smokers and ex-smokers: Reactions of college students to the Surgeon General's cancer report
}

\author{
Martin Katahn and Charles D. Spielberger, VANDERBILT UNIVERSITY \\ Abe J. Judson, UTICA COLLEGE OF SYRACUSE UNIVERSITY
}

\begin{abstract}
Abstraet
A larger proportion of natural science majors, compared with liberal arts majors, quit smoking as a consequence of the report. Smokers questioned the validity of the report, yet rated themselves as more likely to get lung cancer. Approximately half the smokers were dissatisfied with the habit and reported making unsuccessful attempts to quit. Ex-smokers tended to have longer time perspectives than smokers.

\section{Problem}

There seems to be considerable evidence that the probability of developing lung cancer is increased by cigarette smoking. This evidence was recently set forth and widely publicized in the report of the U. S. Surgeon General on January 11, 1964. The goals of the present study were to examine some of the characteristics of college students who stopped smoking and those who did not following this report.
\end{abstract}

\section{Method and Procedure}

The Ss were 394 undergraduates, 253 males and 141 females, at Vanderbilt University and Utica College of Syracuse University. All of the Ss completed a biographical-smoking questionnaire and a Story Completion Test (Barndt \& Johnson, 1955) during regular class periods 8-10 weeks after publication of the report. On the Story Completion Test, Ss were asked to make up two stories, and to indicate the amount of time these stories would have taken to occur in reality. Each story was scored for time perspective on the 6-point scale described by Barndt and Johnson.

\section{Results and Diseussion}

Of the 394 students, $187(47.2 \%)$ reported that they were or had been cigarette smokers. Of the students who identified themselves as smokers, 30 (16.0\%) reported that they had quit smoking prior to the Surgeon General's report for non-cancer related reasons, 141 $\mathbf{( 7 5 . 4 \% )}$ stated that they were continuing to smoke cigarettes, and only $16(8.6 \%)$ indicated that they had quit specifically because of concern over cancer generated by the report. This article is concerned only with those students presently smoking and those who quit because of the cancer report. Both of these groups, to be referred to as smokers and ex-smokers, indicated that their consumption of cigarettes prior to the Surgeon General's report had been, on the average, approximately one pack daily.

Figure 1 presents the percentage of smokers and exsmokers according to sex and college major. Although a slightly higher percentage of males than females reported that they had quit smoking because of the cancer report $(10.3 \%$ to $5.9 \%)$, this difference was not signifi-

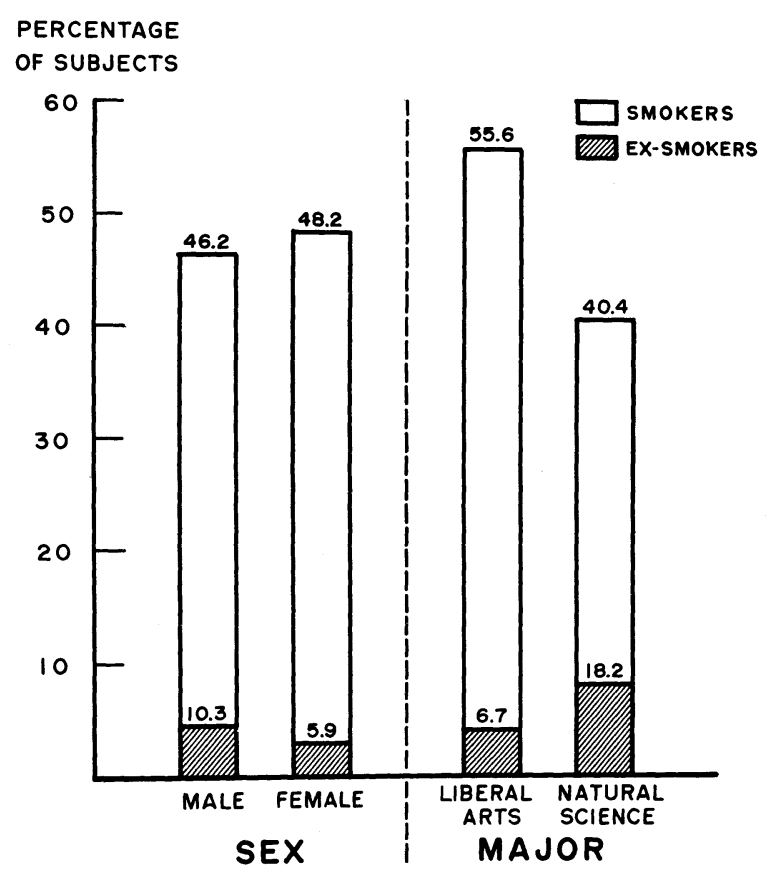

Fig. 1. Percentage of smokers and ex-smokers by sex and college major. The percentage of ex-smokers is drawn in proportion to the size of the group being considered.

cant. Of the students with majors in the natural sciences (including engineering), $40.4 \%$ were smokers compared with $55.6 \%$ of students in liberal arts (including the social sciences). This difference was statistically significant ( $\left.x^{2}=6.78 ; \mathrm{p}<.01\right)$ and consistent with previous findings (Heath, 1958). It would appear that personality differences associated with the choice of a major area are also related to the development of the smoking habit.

Moreover, as can be seen from Fig. 1, a significantly larger percentage of engineering and natural science majors (18.2\%) reported they quit smoking because of their concern over cancer than was the case for liberal arts and social science majors (6.7\%). The $x^{2}$ for this relationship was $4.50 ; \mathrm{p}<.05$. Thus, students with background in the natural sciences were more likely than non-science majors to be influenced by the scientific information conveyed in the Surgeon General's report.

Since the possibility of lung cancer as a consequence of smoking lies in the remote future, it was hypothesized that ex-smokers would characteristically think in terms 
Table 1. Mean Ranks Assigned to Reasons for Smokinga

$$
\begin{array}{ll}
\text { Mean } & \text { Mean } \\
\text { Male } & \text { Female } t
\end{array}
$$

1. It helps me feel relaxed when

$\begin{array}{llll}\text { I am worried or tense (3) } & 1.41 & 1.64 & 2.11^{*}\end{array}$

2. It facilitates sociability and friendship (5)

$2.90 \quad 2.51 \quad 2.17 *$

3. It facilitates thinking and helps when I need inspiration (4)

4. It serves as companionship when I am alone (1)

5. It helps me feel warmer if I am cold (2)

4.34

aPhanishayi's rankings are in parenthesis. The items were rephrased.

*Difference significant at the .05 level.

of a larger time context than smokers. Ex-smokers tended to obtain higher time scores on the Story Completion Test, indicating longer time perspective, than smokers (t, 1.85; df, 155; $\mathrm{p}<.10$ ).

The students were asked to rank order the importance of the five reasons for smoking listed in Table 1 . These items were given by Indian college students in a study by Phanishayi (cited in Matarazzo \& Saslow, 1960) as the most important reasons for their smoking. In the present study, smokers and ex-smokers did not differ in the average ranking of the individual items. Tension reduction was ranked by both men and women as the most important reasons for smoking, and aid to sociability and friendship was second in importance. Except for a reversal of items 3 and 4, the rank order of the reasons was essentially the same for both sexes. However, the mean rank assigned to the tension reducing properties of smoking was significantly higher for males than females, whereas females ranked aid to sociability and friendship significantly higher than males. It is interesting to note that the rank order of reasons for smoking obtained in the present study correlated negatively (rho, -.60) with that given by Phanishayi's Indian students. Although the format of the present test differed from Phanishayi's, the contra- dictory results indicate that the relative importance of reasons for smoking may vary across cultures.

The students rated the extent of their agreement with (acceptance of) the Surgeon General's report on a 5point scale ranging from strongly agree to strongly disagree. Ex-smokers indicated stronger agreement with the report than smokers $(t, 2.94 ; p<.01)$. However, on an 8-point scale varying from 1 in 10 to 1 in $1,000,000$, the smokers rated themselves as much more likely to get lung cancer than did ex-smokers (approximately 1 chance in 500 as compared with 1 in 5,000; $\mathrm{t}, 2.62 ; \mathrm{p}<.01)$. Thus, the smokers indicated less acceptance of the report than the ex-smokers, yet gave themselves a significantly greater likelihood of getting cancer. This paradox suggests that many smokers, while disturbed by the lung cancer reports, have been unable or unwilling to give up the habit.

In this connection, widespread discontent with smoking was indicated by $44.7 \%$ of the smokers, who rated themselves as either moderately or vary dissatisfied with the habit. Furthermore, nearly half of the smokers $(47.5 \%)$ reported they had made one or more attempts to quit during the past year. This expressed dissatisfaction with on-going behavior suggests an underlying conflict. Here is a habit, engaged in primarily for the purpose of tension reduction, which itself generates anxiety because of its potentially dangerous effects. It would appear that in refusing to accept the validity of the Surgeon General's report, the smokers in this study were attempting to repress or deny their fears about the consequences of smoking. Acceptance of the report would intensify their conflict and increase the general tension they seek to reduce by smoking. That their defenses are not completely effective is indicated by their acceptance of a higher probability of developing lung cancer.

\section{References}

BARNDT, R. J., \& JOHNSON, D. Time orientation in delinquents. J. abn. soc. Psychol., 1955, 51, 343-345.

HEATH, C. W. Differences between smokers and nonsmokers. AMA Arch. intern. Med., 1958,101,377-388. MATARAZZO, J. D., \& SASLOW, G. Psychological and related characteristics of smokers and nonsmokers. Psychol. Bull., 1960, 57, 493-513.

\begin{abstract}
PAVLIK, W. B., \& CARLTON, P. L. (Rutgers U., New Brunswick, N. J.). The effects of partial and continuous reinforcement schedules were compared on both a between and a within subjects basis. Rats given either partial or continuous reinforcement in a lever pressing situation (between subjects comparison) showed the usual partial reinforcement effects; both greater resistance to extinction and higher terminal
\end{abstract}

acquisition response rates were associated with partial reinforcement. The same pattern of results did not, however, appear when a within subjects comparison was made. Rats exposed to both continuous and partial reinforcement schedules showed higher terminal acquisition performance in the partial reinforcement condition, but showed greater resistance to extinction in the continuous reinforcement condition. (Pre-publication copies are available from the first author.) 